\title{
EFECTO DE LAS DENSIDADES DE SIEMBRA Y UN FUNGICIDA EN EL RENDIMIENTO DEL FRIJOL ${ }^{1}$
}

\author{
Bernardo Mora $^{2}$, Santiago Fernández ${ }^{2}$, Guillermo Flores ${ }^{2}$, Arturo Solórzano ${ }^{2}$
}

\section{RESUMEN}

Efecto de las densidades de siembra y un fungicida en el rendimiento del frijol. En cinco localidades de Costa Rica se evaluaron las variedades de frijol Guaymí y la línea MUS 181 en cinco densidades de siembra (140.000, 170.000, 200.000, 230.000 y 260.000 plantas por hectárea), con y sin aplicación de fungicida Benomy1 50 PM en dosis de 0,35 $\mathrm{kg} / \mathrm{ha}$. Se determinaron las pérdidas en rendimiento causadas por patógenos y se realizó un análisis económico de la actividad. Hubo baja incidencia de Mustia Hilachosa y las densidades de siembra de 140.000 a 170.000 plantas por hectárea presentaron los mayores beneficios netos y la más alta tasa de retorno marginal.

\begin{abstract}
Effect of plant population density and fungicide application on bean yield. Varieties of Guaymí bean and the MUS 181 line were evaluated in five locations in Costa Rica, in five plant populations $(140.000 ; 170.000 ; 200.000$; 230.000 and 260.000 plants per hectare). Some plants were sprayed with benomyl 50PM fungicide at $0.35 \mathrm{~kg}$ per hectare, while others did not receive such treatment. Yield losses caused by pathogenic agents were determined, and an economic analysis of the treatment was performed. A low incidence of Mustia Hilachosa/bean diseases was reported on treated plants. Plant populations of 140.000 and 170.000 plants per hectare produced the greatest profit and the highest marginal return rateo
\end{abstract}

\section{INTRODUCCIÓN}

La mustia hilachosa (Rhizoctonia solani Kühn = Thanatephorus cucumeris Frank) del frijol, conjuntamente con otras enfermedades y plagas, constituye una fuerte limitante en la producción de este cultivo, en las diversas regiones frijoleras del país (Cardozo; Oliveira, 1982; Echandi, 1966; Galindo; Abawi; Thurston, 1982; Galindo; Abawi; Thurston, 1983; Mora, 1987; Mora; Villalobos; Gálvez et al. 1989; Quesada et al., 1992). El hongo ataca en cualquier estado de desarrollo de la planta, y en ausencia de algún tipo de manejo de la enfermedad, las pérdidas en rendimiento son de considerable importancia económica (Cardozo, 1980; Cardozo; Oliveira, 1982; Echandi, 1965; Echandi, 1966; Galindo et al. 1982; Limkemer, 1986; Manzano, 1973; Mora, 1987; Mora, et al. 1989; Rosado; García, 1985; Zaumeyer; Thomas, 1957).

El estudio poblacional en cualquier cultivo, debe hacerse para cada variedad de acuerdo a su arquitectura, zona y época de siembra (Galindo; Abawi; Thurston, 1983; Gálvez; Guzman; Castaño, 1979; Joyce; Berg- gren; Berner, 1990; Lewis; Papavizas, 1980; Prabhu et al., 1983; Prabhu et al., 1982). Además, es recomendable que se realice en varias épocas de siembra y durante varios años, para obtener resultados confiables que puedan ser recomendados a los agricultores. Las densidades de siembra que se recomienda en las diferentes zonas y sistemas de cultivo son prácticamente las mismas. Debido a lo anterior es imperativo investigar la densidad de siembra en el cultivo de acuerdo a la variedad, manejo, zona y época de siembra. La población de plantas por hectárea que utilizan los agricultores en las diferentes regiones frijoleras del país, obedece más a una escogencia propia de ellos, que a una recomendación previamente investigada y validada a escala regional. Los agricultores de las zonas donde se hizo el presente trabajo utilizan densidades de plantas que van de 92.000 a 180.000 plantas por hectárea.

El objetivo del trabajo fue determinar el efecto de las densidades de siembra en frijol, con y sin aplicación de fungicida. Además de medir el impacto económico de estos tratamientos.

1 Trabajo financiado por el Programa Cooperativo Regional de Frijol de Centroamérica, México y el Caribe (PROFRIJOL) .

2 Dirección de Investigaciones Agropecuarias, Ministerio de Agricultura y Ganadería, Apdo. 10094, San José: Costa Rica 


\section{MATERIALES Y MÉTODOS}

Los trabajos se realizaron en Veracruz de Pejibaye de Pérez Zeledón; en la Estación Experimental Fabio Baudrit (EEFB), localizada en el Barrio San José de Alajuela; en Nances de Esparza; en Llano Azul de Canalete de Upala y en Pavón de Los Chiles. Los experimentos se ubicaron en fincas comerciales con excepción de la estación experimental, bajo el sistema de siembra usada por los agricultores de la región, caracterizado, en unos por la mínima labranza y en otros por un manejo convencional del suelo por medio de maquinaria agrícola.

El diseño experimental que se utilizó fue de parcelas sub-sub divididas para variedades y aplicación o no de fungicidas. Las desidades de siembra constituyeron la parcela pequeña y se distribuyeron internamente, como bloques completos al azar. El modelo utilizado en este diseño se presenta como: Yijk1 $=\mathrm{U}+\mathrm{Bi}+\mathrm{Vj}+$ $B V i j+F k+V F j k+B F(v) i j k+D 1+V D j 1+D F l k$ VDFjlk + BDFDllkl

La distancia entre hileras utilizada en todos los experimentos fue de $50 \mathrm{~cm}$. Las densidades que se evaluaron fueron $140.000,170.000,200.000,230.000 \mathrm{Y}$ 260.000 plantas por hectárea. Las siembras que se realizaron en Pejibaye, EEFB y Esparza se hicieron a espeque, colocando de dos a tres semillas por hoyo. A la siembra se aplicó la fórmula comercial de fertilizante 10-30-10 a razón de $200 \mathrm{~kg} / \mathrm{ha}$. Se utilizó un caracolicida para evitar el ataque de las babosas y como insecticida de suelo se aplicó Foxim en dosis de $10 \mathrm{~kg} / \mathrm{ha}$. El control de malezas se hizo con pendimentalina a razón de $1 \mathrm{~kg} / \mathrm{ha}$.

En la localidad de Upala y Los Chiles los surcos se trazaron con un rayador accionado por tractor. En ambos sitios la fertilización se hizo al momento de la siembra. El control de malezas se realizó con pendimentalina en preemergencia al cultivo de frijol y en postemergencia se utilizó el bentazón y fluazifop butil, a razón de 1,0 gi.a./ha de producto comercial en ambos casos.

Los materiales de frijol que se utilizaron fueron la variedad Guaymí (MUS 106) y la línea MUS 181; ambas de resistencia intermedia a mustia hilachosa. Los dos cultivares son de hábito II b erecto y grano de color negro. El cultivar MUS 181 se caracteriza por ser erecto y producir sus vainas cerca del eje principal de la planta, mientras que la variedad Guaymí produce una mayor ramificación y tiende a ser más postrada enm hábito de crecimiento.

Las dos aplicaciones de benomyl (50\% PM) en los todos los experimentos, se realizaron a los 20 y 40 días des- pués de la siembra, en dosis de $0,35 \mathrm{~kg} / \mathrm{ha}$. No fue necesaria la aplicación de insecticidas para combatir insectos de follaje, ya que el nivel de daño fue inferior al $3 \%$.

El análisis estadístico de los resultados se realizó por medio del SAS (Statistical Analysis System). En todos los experimentos se analizaron el número de plantas cosechadas por parcela, el número de vainas en cinco plantas y el rendimiento expresado en gramos por parcela. Además, se realizó una correlación simple entre las variables estudiadas y la densidad con el objetivo de observar la tendencia de los tratamientos.

\section{RESULTADOS Y DISCUSIÓN}

Todas las siembras comerciales que se realizaron durante el año 1996, en las diversas regiones frijoleras de Costa Rica fueron afectadas por el exceso de precipitación, lo que trajo grandes pérdidas en el rendimiento en algunas zonas. Los ensayos que se realizaron en la Estación Experimental Fabio Baudrit (EEFB) y en Los Chiles fueron los que sufrieron más por el estrés causado por el exceso de la lluvia, lo cual se refleja en un menor rendimiento.

El análisis de variación (Andeva), para los datos de rendimiento, obtenidos en Veracruz de Pejibaye de Pérez Zeledón, no mostró diferencia significativa en cuanto a variedades, aplicación de fungicidas, densidades, y sus interacciones. Las variables de número de plantas cosechadas y número de vainas por plantas sí mostraron diferencia estadística significativa. Los datos de rendimiento en Esparza presentaron diferencia estadística entre los materiales de frijol, la línea MUS 181 rindió más que la variedad Guaymí. Las otras dos variables analizadas también presentaron diferencia estadística significativa.

Los datos de rendimiento y número de plantas cosechadas obtenidos en la, EEFB, mostraron diferencia significativa para variedades y densidades, no así para el número de vainas por planta. En el ensayo de Los Chiles hubo diferencias en rendimiento por efecto de las densidades, mientras que en Upala hubo diferencias en rendimiento por la aplicación de fungicidas y entre los materiales de frijol. En ambas localidades las otras dos variables en estudio también presentaron diferencia significativa.

En el Cuadro 1 se presenta el rendimiento y sus componentes evaluados en Guaymí y MUS 181, en los diversos lugares que se realizaron los experimentos. Los resultados se sumarizan en un mismo cuadro, no obstante, el objetivo del estudio no fue comparar el rendimiento y sus componentes entre las regiones, sino 
Cuadro 1. Promedios de rendimiento (Rend.) en gramos por parcela útil, plantas cosechadas (Pl. Cos.) y vainas por planta (Vn. Pl.), en dos variedades de frijol sembradas en diversas zonas frijoleras de Costa Rica. 1996-1997.

\begin{tabular}{|c|c|c|c|c|c|c|}
\hline \multirow{3}{*}{ Localidades } & \multicolumn{6}{|c|}{ Cultivares } \\
\hline & \multicolumn{3}{|c|}{ Guaymí } & \multicolumn{3}{|c|}{ MUS 181} \\
\hline & Rend. & Pl. Cos. & V n. PI. & Rend. & Pl. Cos. & $\begin{array}{l}\text { Vn.PI } \\
\end{array}$ \\
\hline Pérez Zeledón $(6,0 \mathrm{~m})^{1}$ & $1214 \mathrm{a}^{2}$ & $113 \mathrm{a}$ & $8,6 \mathrm{a}$ & $1186 \mathrm{a}$ & $108 \mathrm{a}$ & $8,8 a$ \\
\hline Alajuela $(4,5 \mathrm{~m})$ & $391 \mathrm{a}$ & $94 \mathrm{a}$ & $6,8 \mathrm{a}$ & $481 \mathrm{a}$ & $100 \mathrm{a}$ & $6,4 a$ \\
\hline Esparza $(4,5 \mathrm{~m})$ & $900 \mathrm{a}$ & $77 \mathrm{a}$ & $11,0 \mathrm{a}$ & $982 \mathrm{a}$ & $79 \mathrm{a}$ & $9,6 a$ \\
\hline Upala (4,5 m) & $765 \mathrm{~b}$ & $85 \mathrm{a}$ & $12,8 \mathrm{a}$ & $846 \mathrm{a}$ & $91 \mathrm{a}$ & $12,2 \mathrm{a}$ \\
\hline Los Chiles $(6,0 \mathrm{~m})$ & $606 a$ & $111 \mathrm{a}$ & $8,8 \mathrm{~b}$ & $636 \mathrm{a}$ & $105 \mathrm{~b}$ & $9,5 a$ \\
\hline
\end{tabular}

1 Area de parcela útil cosechada

2 Promedio con igual letra no son significativamente diferentes, de acuerdo a la prueba de Tukey $(\mathrm{p}<=0,05)$.

estudiar dentro de cada región, la respuesta de los materiales de frijol con las diferentes prácticas de manejo. La diferencia estadística debe considerarse entre los dos cultivares sembrados en una misma localidad.

En rendimiento, sólo existió diferencia significativa en el ensayo realizado en Upala, entre los materiales Guaymí y MUS 181. En las otras localidades no hubo diferencia estadística. En cuanto a la variable de vainas por planta hubo diferencia significativa sólo en el experimento realizado en Los Chiles.

En el Cuadro 2 se presentan los promedios de rendimiento para las variables estudiadas, con y sin aplicación de fungicida en las diferentes localidades. Nótese que dentro de las localidades al igual que en el cuadro anterior, existió diferencia estadística en cuanto a rendimiento en la región de Upala y en cuanto al número de vainas por planta en la localidad de Los Chiles.
La diferencia en el experimento de Upala se debió a una mayor severidad de mustia hilachosa en las subparcelas sin aplicación de benomyl, lo que provocó un menor rendimiento a pesar de que ambas variedades son de resistencia intermedia al hongo. La diferencia estadística en el experimento de Los Chiles en el número de vainas por planta, se debió a que algunas plantas tuvieron un menor desarrollo debido a una mayor compactación producida por el tractor al marcar el terreno.

En el Cuadro 3 se muestran las medias para el rendimiento, en las diversas localidades con las diferentes densidades de siembra. Observe que los resultados en cuanto a rendimiento de Pérez Zeledón, Esparza y Upala no presentaron diferencia estadística. Este resultado indica que poblaciones menores rindieron de forma similar a poblaciones mayores de plantas. Esto podría explica también el por qué los agricultores se inclinan más a utilizar poblaciones menores de plantas, ya que van

Cuadro 2. Promedios de rendimiento (Rend.) en gramos por parcela útil, plantas cosechadas (Pl. Cos.) y vainas por planta (Vn. Pl.), en dos variedades de frijol con y sin aplicación de fungicida en cinco localidades de Costa Rica. 1996-1997.

\begin{tabular}{|c|c|c|c|c|c|c|}
\hline \multirow[t]{2}{*}{ Localidades } & \multicolumn{3}{|c|}{ Guaymí } & \multicolumn{3}{|c|}{ MUS 181} \\
\hline & Rend. & Pl. Coso & Vn. PI. & Rend. & PI. Cos. & Vn. PI \\
\hline Pérez Zeledón $\left(6,0\right.$ m) ${ }^{1}$ & $1227 \mathrm{a}^{2}$ & $107 \mathrm{~b}$ & $9,2 \mathrm{a}$ & $1173 \mathrm{a}$ & $114 \mathrm{a}$ & $8,2 \mathrm{a}$ \\
\hline Alajuela $(4,5 \mathrm{~m})$ & $435 \mathrm{a}$ & $97 \mathrm{a}$ & $6,6 \mathrm{a}$ & $438 \mathrm{a}$ & $97 \mathrm{a}$ & $7,4 \mathrm{a}$ \\
\hline Esparza (4,5 m) & $934 \mathrm{a}$ & $78 \mathrm{a}$ & $10,0 \mathrm{a}$ & $948 \mathrm{a}$ & $78 \mathrm{a}$ & $10,6 \mathrm{a}$ \\
\hline Upala $(4,5 \mathrm{~m})$ & $847 \mathrm{~b}$ & $86 \mathrm{a}$ & $12,5 \mathrm{a}$ & $764 \mathrm{a}$ & $90 \mathrm{a}$ & $12,5 \mathrm{a}$ \\
\hline Los Chiles $(6,0 \mathrm{~m})$ & $629 a$ & $106 \mathrm{a}$ & $9,4 \mathrm{a}$ & $612 \mathrm{a}$ & $110 \mathrm{~b}$ & $8,8 \mathrm{~b}$ \\
\hline
\end{tabular}

${ }^{1}$ Area de parcela útil cosechada

2 Promedio con igual letra no son significativamente diferentes, de acuerdo a la prueba de Tukey $(\mathrm{p}<=0,05)$. 
Cuadro 3. Promedios de rendimiento en gramos por parcela útil, de dos variedades de frijol con y sin aplicación de fungicida, sembradas en diferentes densidades de siembra en diversas regiones frijoleras de Costa Rica. 1996-1997.

\begin{tabular}{lccccc}
\hline Densidades & $\begin{array}{c}\text { Pérez } \\
\text { Zeledón }\end{array}$ & $\begin{array}{c}\text { Alajuela } \\
\text { (EEFB) }\end{array}$ & Esparza & Upala & Los Chiles \\
\hline 260.000 & $1310 \mathrm{a}$ & $501 \mathrm{a}$ & $952 \mathrm{a}$ & $801 \mathrm{a}$ & $578 \mathrm{~b}$ \\
230.000 & $1185 \mathrm{a}$ & $507 \mathrm{a}$ & $977 \mathrm{a}$ & $832 \mathrm{a}$ & $726 \mathrm{a}$ \\
200.000 & $1197 \mathrm{a}$ & $409 \mathrm{ab}$ & $952 \mathrm{a}$ & $791 \mathrm{a}$ & $617 \mathrm{ab}$ \\
170.000 & $1142 \mathrm{a}$ & $455 \mathrm{ab}$ & $893 \mathrm{a}$ & $818 \mathrm{a}$ & $575 \mathrm{~b}$ \\
140.000 & $1172 \mathrm{a}$ & $309 \mathrm{~b}$ & $931 \mathrm{a}$ & $784 \mathrm{a}$ & $605 \mathrm{ab}$ \\
Area parc. $\left(\mathrm{m}^{2}\right)$ & $6,0 \mathrm{~m}$ & $4,5 \mathrm{~m}$ & $4,5 \mathrm{~m}$ & $4,5 \mathrm{~m}$ & $6,0 \mathrm{~m}$ \\
Coefic. Corr. & 0,78 & 0,84 & 0,63 & 0,38 & 0,24 \\
\hline
\end{tabular}

Medias con la misma letra dentro de una misma columna, no son diferentes significativamente de acuerdo a la prueba de Tukey $(\mathrm{p} \leq=0,05)$.

asociadas al menor gasto de insumos y economía en tiempo. Además, un menor número de plantas por hectárea disminuye el índice de área foliar, que en la mayoría de los casos es sustrato para una mayor intensidad de las diversas enfermedades. Los resultados de rendimiento obtenidos en la EEFB y Los Chiles sí mostraron significancia en las diferentes densidades. Los análisis de correlación simple entre densidad y rendimiento en gramos por parcela mostraron valores aceptables en los experimentos de Pérez Zeledón y EEFB. En Esparza el valor de la correlación fue intermedia y en el caso de Upala y Los Chiles el valor de la correlación fue baja.

En el Cuadro 4 se presenta el promedio de plantas cosechadas con los materiales y las densidades de siembra en las diferentes regiones. El número de plantas por parcela en cada una de las densidades se estableció dos semanas después de la siembra. La diferencia estadística es inducida al prefijar las poblaciones. En la siembra de Los Chiles la población de plantas disminuyó debido al ataque de Sclerotium rolfsii. En Upala, Esparza y Pérez Zeledón la cantidad de plantas fue menor debido al ataque de Rhizoctonia solani y en la EEFB se debió al ataque de Fusarium oxysporum f.s. phaseoli. Debido a lo anterior se realizó la prueba de diferenciación de medias, sin embargo, en localidades como Esparza no se dio la diferencia inducida y algunas medias se homogenizaron, debido a la mayor o menor pérdida de plantas.

Los coeficientes de correlación entre las densidades de siembra y las plantas cosechadas fueron arriba de 0,92 aunque el esperado era de uno. Lo anterior se debió a que después que se fijaron las poblaciones de plantas, algunas murieron por problema de plagas y enfermedades.

Cuadro 4. Promedios de plantas cosechadas en dos variedades de frijol con y sin aplicación de fungicida, sembradas en diferentes densidades de siembra en diversas regiones frijoleras de Costa Rica. 1996-1997.

\begin{tabular}{lccccc}
\hline \multicolumn{1}{c}{ Densidades } & $\begin{array}{c}\text { Pérez } \\
\text { Zeledón }\end{array}$ & Alajuela & Esparza & Upala & Los Chiles \\
\hline 260.000 & $140 \mathrm{a}$ & $117 \mathrm{a}$ & $86 \mathrm{a}$ & $108 \mathrm{a}$ & $124 \mathrm{a}$ \\
230.000 & $123 \mathrm{~b}$ & $107 \mathrm{ab}$ & $85 \mathrm{a}$ & $97 \mathrm{ab}$ & $127 \mathrm{a}$ \\
200.000 & $111 \mathrm{c}$ & $103 \mathrm{~b}$ & $84 \mathrm{a}$ & $86 \mathrm{~b}$ & $107 \mathrm{~b}$ \\
170.000 & $97 \mathrm{~d}$ & $86 \mathrm{~b}$ & $71 \mathrm{~b}$ & $85 \mathrm{~b}$ & $97 \mathrm{bc}$ \\
140.000 & $82 \mathrm{e}$ & $72 \mathrm{c}$ & $64 \mathrm{~b}$ & $67 \mathrm{c}$ & $85 \mathrm{bc}$ \\
Area parc $\left(\mathrm{m}^{2}\right)$ & $6,0 \mathrm{~m}$ & $4,5 \mathrm{~m}$ & $4,5 \mathrm{~m}$ & $4,5 \mathrm{~m}$ & $6,0 \mathrm{~m}$ \\
Coefic. Corr. & 0,99 & 0,98 & 0,92 & 0,97 & 0,95 \\
\hline
\end{tabular}

Medias con la misma letra no son significativamente de acuerdo a la prueba de Tukey $(\mathrm{p}<=0,05)$. 
En todas las localidades existió la tendencia a incrementarse el número de vaina en las siembras de menor densidad (Cuadro 5). Lo anterior se debe a que a menor densidad de siembra el número de vainas tiende a incrementarse ya que la planta tiene mayor espacio, para competir por luz y nutrimentos. Los valores de los coeficientes de correlación entre densidades y número de vainas por planta, fueron inversamente proporcionales y con valores significativos en las localidades de Pérez Zeledón y la EEFB. En los experimentos de Upala y Los Chiles, la correlación fue intermedia, mientras que en el experimento de Esparza el valor de la correlación fue proporcionalmente positiva con un valor de 0,67 .

Estos ensayos se realizaron bajo el criterio de manejo integrado del cultivo, donde las actividades se implementaron en el sistema de siembra que utilizan los agricultores en cada una de la regiones. El manejo fitosanitario en los experimentos se realizó con un criterio de umbral económico, con el objetivo de buscar rentabilidad, sin hacer un uso excesivo de los insumos agrícolas.

En el experimento de Pejibaye de Pérez Zeledón el mayor problema al momento de la siembra fue la babosa , la cual se redujo con dos aplicaciones de un caracolicida a base de metaldehído, una a la siembra y otra dos semanas después. Las enfermedades más importantes fueron mustia hilachosa y mancha angular (Phaeoisariopsis griseola), sin embargo los niveles de infección no fueron significativos, ya que la severidad evaluada en promedio fue inferior al $5 \%$.

En la siembra realizada en Alajuela (EEFB) el mayor problema fue Fusarium oxysporum f.s. phasoli que disminuyó la población de la mayoría de las parcelas de la primera repetición del ensayo. En el experimento de
Esparza se tuvo que eliminar una repetición completa ya que el ataque de joboto o gallina ciega (Phyllophaga spp.) afectó todos los tratamientos de una subparcela. En el ensayo de Los Chiles fue el ataque de Sclerotrium rolfsii tal como se comentó anteriormente. No obstante, el ataque fue uniforme y en bajo porcentaje de ahíque los tratamientos no se afectaron significativamente.

Los programas de mejoramiento genético, en la mayoría de los casos liberan variedades para dar respuestas a limitantes asociadas a características genotípicas. No obstante, siendo el fenotipo una interacción entre germoplasma y medio ambiente, es necesario evaluar el material promisorio bajo diversas estrategias de manejo agronómico, donde se incluya época de siembra, densidad y arreglo topológico. Además, de pruebas de fertilización, manejo sanitario y práctica de post-cosecha.

Cuando se trabaja con variedades que presentan resistencia intermedia a R. solani, existe oportunidad para que otras enfermedades se presenten con mayor intensidad. Debido a lo anterior, es recomendable trabajar con el complejo general de enfermedades del cultivo y valorar la mayor o menor intensidad de cada una de ellas. Además, es recomendable evaluar la incidencia de insectos de suelo y follaje, así como el manejo de malezas.

En el Cuadro 6 se presenta el resumen sobre análisis de dominancia (CIMMYT, 1988) de las diferentes densidades de siembra, por cultivar con y sin fungicida según localidad. Se puede observar que el cultivar MUS 181 sin fungicida y a densidades bajas obtuvo los beneficios netos más altos y la tasa de retorno marginal mayor. No obstante en la localidad de Llano Azul, Upala, se observó un efecto de la aplicación de fungicida

Cuadro 5. Promedio del número de vainas por planta en dos variedades de frijol con y sin aplicación de fungicida, sembradas en diferentes densidades de siembra en diversas regiones frijoleras de Costa Rica. 1996-1997.

\begin{tabular}{lccccc}
\hline \multicolumn{1}{c}{ Densidades } & $\begin{array}{c}\text { Pérez } \\
\text { Zeledón }\end{array}$ & Alajuela & Esparza & Upala & Los Chiles \\
\hline 260.000 & $38 \mathrm{c}$ & $31 \mathrm{a}$ & $50 \mathrm{~b}$ & $54 \mathrm{~b}$ & $44 \mathrm{a}$ \\
230.000 & $39 \mathrm{c}$ & $30 \mathrm{a}$ & $49 \mathrm{~b}$ & $66 \mathrm{a}$ & $44 \mathrm{a}$ \\
200.000 & $42 \mathrm{bc}$ & $33 \mathrm{a}$ & $46 \mathrm{~b}$ & $65 \mathrm{a}$ & $49 \mathrm{a}$ \\
170.000 & $47 \mathrm{~b}$ & $34 \mathrm{a}$ & $51 \mathrm{~b}$ & $63 \mathrm{ab}$ & $46 \mathrm{a}$ \\
140.000 & $51 \mathrm{a}$ & $37 \mathrm{a}$ & $63 \mathrm{a}$ & $64 \mathrm{a}$ & $46 \mathrm{a}$ \\
Area parc. $\left(\mathrm{m}^{2}\right)$ & $6,0 \mathrm{~m}$ & $4,5 \mathrm{~m}$ & $4,5 \mathrm{~m}$ & $4,5 \mathrm{~m}$ & $6,0 \mathrm{~m}$ \\
Coefic. Corr. & $-0,97$ & $-0,92$ & 0,67 & $-0,55$ & $-0,46$ \\
\hline
\end{tabular}

Medias con la misma letra no son significativamente de acuerdo a la prueba de Tukey $(\mathrm{p}<=0,05)$. 
Cuadro 6. Densidad de siembra, ingreso total, costo variable, beneficio neto y tasa retorno marginal (TRM) de dos cultivares de frijol negro con y sin fungicida de acuerdo a su dominancia, según localidad, Costa Rica. 1996-1997. ${ }^{1}$

\begin{tabular}{lllrrrrr}
\hline Localidad & Variedad & $\begin{array}{c}\text { Control } \\
\text { enfermedad }\end{array}$ & $\begin{array}{c}\text { Densidad miles } \\
\text { plantas/ha }\end{array}$ & $\begin{array}{c}\text { Ingreso } \\
\text { colon/ha }\end{array}$ & $\begin{array}{c}\text { Costo } \\
\text { variable/ha }\end{array}$ & $\begin{array}{c}\text { Beneficio } \\
\text { neto/ha }\end{array}$ & $\begin{array}{c}\text { Tasa reto } \\
\text { marg. (\%) }\end{array}$ \\
\hline Pejibaye & Guaymí & Sin fung & 140 & 262.265 & 44.902 & 217.363 & \\
(Pérez Zeledón) & Mus 181 & Sin fung & 140 & 308.144 & 48.028 & 60.117 & 1.368 \\
La Garita & Guaymí & Sin fung & 140 & 88.715 & 48990 & 39.725 & \\
(Alajuela) & Mus 181 & Sin fung & 140 & 105.606 & 51.390 & 54.216 & 604 \\
& Guaymí & Sin fung & 170 & 121.432 & 58.680 & 62.752 & 117 \\
& Mus 181 & Sin fung & 170 & 154.087 & 60.930 & 93.157 & 1.351 \\
Nances & Guaymí & Sin fung & 140 & 276.341 & 44.665 & 231.676 & \\
(Esparza) & Mus 181 & Sin fung & 140 & 296.732 & 47.065 & 249.667 & 750 \\
Llano Azul & Guaymí & Sin fung & 140 & 218.212 & 52.590 & 165.622 & 182 \\
(Upala) & Mus 181 & Sin fung & 140 & 237.385 & 54.790 & 182.595 & 772 \\
& Mus 181 & Con fung & 140 & 274.819 & 71.949 & 202.870 & 118 \\
Pavón & Mus 181 & Con fung & 170 & 291.862 & 78.764 & 213.098 & 150 \\
(Los Chiles) & Mus 181 & Sin fung & 140 & 137.866 & 48.290 & 89.576 & 34.138 \\
\hline
\end{tabular}

11 US $\$=214.66$ colones.

Fuente: Guillermo Flores M., Area Socioeconomía DlA-MAG, con base en información suministrada por los agricultores de las diferentes localidades. 1997.

debido a que hubo una mayor severidad en el ataque de mustia hilachosa y mancha angular (Flores, 1997).

En el trabajo realizado en la Estación Experimental Fabio Baudrit en Alajuela el mayor beneficio neto y la tasa de retorno marginal se obtuvo con la densidad de 170.000 planta/ha, debido al manejo agronómico utilizado en esta localidad (Flores, 1997).

Los datos obtenidos en cada una de las localidades sugiere al productor que la inversión en el cambio tecnológico por adoptar un nuevo cultivar, distancia de siembra o aplicación de fungicida le podría ser retribuido bajo las condiciones en que se realizó la siembra (Flores, 1997).

Las variedades utilizadas manifestaron buena resistencia a mustia hilachosa bajo las condiciones en que se presentó la enfermedad.

Una o dos aplicaciones de benomyl50 PM sería una alternativa de manejo bajo condiciones muy justificadas.

Densidades de siembra. de 140.000 a 170.000 plantas por hectárea produjeron los mayores beneficios netos y la mayor tasa de retorno marginal.

\section{LITERATURA CITADA}

CARDOZO, J.E. 1980. Eficiencia de tres fungicidas no controJe da murcha da teia micelica do feijoeiro no Acre. Comunicado Técnico No. 18, 3 p.

CARDOZO, J.E.; DE OLIVEIRA, E.B. 1982. Controle da mela do feijoeiro a través de fungicidas. Pesq. Agropeco Bras. 17:1811-1813.

CENTRO AGRONÓMICO PARA EL MEJORAMIENTO DE MAÍZ Y TRIGO (CIMMYT). 1988. La formulación de recomendaciones a partir de los datos agronómicos. Un manual metodológico de evaluación económica. Rev. México D.E México,CIMMYT. 79p.

ECHANDI, E. 1965. Basidiospore infection by Pellicularia filamentosa (Corticium microsclerotia), the incitant of web bligth of common bean. Phytopathology 55:698699.

ECHANDI, E. 1966. Principales enfermedades del frijol observadas en diferentes zonas eco lógicas de Costa Rica. Turrialba 16:359-363.

FLORES, G. 1997. Análisis económico en los ensayos sobre validación de tecnología en MIP-Mustia en cultivares promisorios de frijol. San José, Costa Rica. Dirección de Investigaciones Agrícolas, Ministerio de Agricultura y Ganadería. 30 p. 
GALINDO, J.J.; ABAWI, G.S.; THURSTON, H.D 1982. Web blight of beans on small fanns in Central America. New York's Food and Life Sciences Quarterly 14:2125.

GALINDO, J.J; ABAWI, G.S.; THURSTON, H.D 1983. Effect of mulching on web bligth of beans in Costa Rica. Phytopathology 73:610-615.

GALINDO, J.J; ABAWI, G.S.; THURSTON, H.D; GÁLVEZ, G.E. 1983. Source of inoculum and development of bean web blight in Costa Rica. Plant Disease 67:1016-1021.

GÁLVEZ, G.E.; GUZMAN, P.; CASTAÑO, M., 1979. Web bligth. Pages 101-110. In: Bean production problems: Disease, Insect, Soil and Climatic Constraints of Phaseolus vulgaris. H. E Schwartz and G.E. Gálvez, eds. Centro Internacional de Agricultura Tropical. Cali, Colombia. 424 p.

JOYCE, G.E; BERGGREN, G.T; BERNER, D.K., 1990. Effects of row spacing and withinrow plant population on Rhizoctonia aerial bligth of soybean and soybean yield. Plant Disease 74: 158-160.

LEWIS, J.A.; PAPAVIZAS, G.C. 1980. Integrated control of Rhizoctonia fruit rot of cucumber. Phytopathology 70:85-89.

LIMKEMER, J. 1986. Efecto de la telaraña (Thanatephorus cucumeris (Frank) Donk= Rhizoctonia solani Kühn sobre el rendimiento de dos cultivares de frijol, bajo el sistema asociado y en relevo con maíz. Tesis de Ing. Agr. Universidad de Costa Rica. San José. 49 p.

MANZANO, J.M. 1973. Evaluación de fungicidas para el control de la mustia hilachosa Thanatephorus cucume- ris y su efecto sobre el cultivo del frijol común en El Salvador. In: XIX Reunión Anual del Programa Cooperativo Centroamericano para el Mejoramiento de los Cultivos Alimenticios. PCCMCA. San José, Costa Rica. pp. 17-30.

MORA, RE., 1987. Manejo integrado de mustia hilachosa en Costa Rica. Seminarios Internos. Centro Internacional de Agricultura Tropical. Cali, Colombia. 10 p.

MORA, B.E.; VILLALOBOS, E; GÁLVEZ, G. 1989. Effect of fungicides on Thanatephorus cucumeris (Frank) Donk, in bean cultivars. Phytopathology 79: 1179. (Abstr.).

PRABHU, A.; SILVA, J.; CORREA, J.; POLARO, R; LIMA, E. 1983. Murcha da teia micelica do feijoeiro comun. Epidemiología e aplicaçao de fungicides. Pesq. Agr. Bras. 12: 1323-1332.

PRABHU, A. S.; POLARO, RH.; VIANA, C.JR; DE ASSIS, F.D.S.J.E; ZIMMERMANN, P.F.J. 1982. Relaçao entre murcha da teia micelica e produçao no feijoeiro comum. Pesq. Agropec. Bras. 17:1607-1613.

QUESADA, A.S; MORA, B.E.; GÁLVEZ, G.E.; ROJAS M.R 1992. Evaluación de fungicidas a base de estaño para el control de Thanatephorus cucumeris en frijol común. Phytopathology 82:610 (abstr.).

ROSADO, F.J.; GARCÍA, R 1985. Incidencia de la mustia hilachosa (Thanatephorus cucumeris) en frijol común, como resultado del manejo del suelo. Rev. Mex. de Fitopatologia. 3:27-34.

ZAUMEYER, W.; THOMAS, H. 1957. A monographic study of bean diseases and methods for their control. U.S. Dep. Agr. Tech. Bull. 868:63-65. 